TRANSACTIONS OF THE

AMERICAN MATHEMATICAL SOCIETY

Volume 362, Number 3, March 2010, Pages 1325-1350

S 0002-9947(09)04939-3

Article electronically published on October 1, 2009

\title{
THE BEHAVIOR OF THE SPECTRAL GAP UNDER GROWING DRIFT
}

\author{
B. FRANKE, C.-R. HWANG, H.-M. PAI, AND S.-J. SHEU
}

\begin{abstract}
We analyze the behavior of the spectral gap of the LaplaceBeltrami operator on a compact Riemannian manifold when a divergencefree drift vector field is added. We increase the drift by multiplication with a large constant $c$ and ask the question how the spectral gap behaves as $c$ goes to infinity. It turns out that the spectral gap stays bounded if and only if the drift-vector field has eigenfunctions in $H^{1}$. In that case the spectral gaps converge and we determine the limit.
\end{abstract}

\section{INTRODUCTION}

1.1. The origins of the problem. In many practical applications from physics, chemistry and engineering it is relevant to understand the motion of a diffusing substance in a fluid flow. Numerous terms such as mixing, stirring, chaotic advection and turbulence are used to describe the mechanisms which determine the ways the diffusion is influenced by the flow (see Ottino [25, Aref [2], Wiggins and Ottino 28, Shraiman and Siggia 27] for some overview articles). Much of the existing work consists of describing simplified models, where stochastic drift describes the chaotic motion of the fluid. This makes sense in view of the difficulty in solving the underlying Navier-Stokes problem explicitly. In the situation of stationary flows however one could hope to use dynamical information from the underlying flow to describe the long-term behavior of the diffusion. Different quantities can be used to describe the influence of the flow on the diffusion. For periodic stationary flows the effective diffusivity and the asymptotic variance are good quantities to study (see Bhattacharya et al. 4, Fannjiang and Papanicolaou [1], Heinze [15]). On compact spaces the diffusion will approach equilibrium when time becomes large. The proximity of the distribution to the equilibrium can be measured in terms of $L^{p}$-norms of the diffusion semigroup for mean-zero elements. It was proved in Franke 12 that those $L^{p}$-norms can be bounded by the $L^{p}$-norms of heat semigroups on suitable comparison manifolds uniformly over the class of divergence-free drift-vector fields.

Received by the editors October 25, 2007.

2000 Mathematics Subject Classification. Primary 35P15, $60 \mathrm{H} 30$.

Key words and phrases. Laplace-Beltrami operator, divergence free drift, spectral gap.

The first author was supported by the DFG, Förderungsnummer: FR2481/1-1.

The second author was supported by the NSC Grant of Republic of China NSC95-2115-M001-012.

The second, third, and fourth authors were partially supported by the Mathematics Division, NCTS (Taipei Office).

The fourth author was supported by the NSC Grant of Republic of China NSC96-2119-M-001002.

(C)2009 American Mathematical Society Reverts to public domain 28 years from publication 
This is however just a worst case analysis and does not give information about the improvement of diffusivity in the presence of a drift. The improvement of the diffusivity through the flow can be measured by increasing the speed of the flow. This is done by multiplication of the drift-vector field with a large constant. The decay of the norms of the diffusion semigroup as the constant becomes large is then due to the mixing properties of the flow. Recently Constantin et al. 9] proved that if the drift-vector field has no $H^{1}$-eigenfunctions, then the $L^{2}$-norm of the diffusion semigroup becomes arbitrarily small when the flow speeds up. We will come back to this result in more detail later. The decay of the semigroup is determined by the spectral gap of the generator of the diffusion. It is therefore useful to understand the behavior of the spectral gap as the constant in front of the drift becomes large. This approach can be found in Berestycki et al. 3. for bounded domains with Dirichlet conditions. Their method is however restricted to the principal eigenvalue of the diffusion generator. This makes sense for the Dirichlet problem, where the decay is determined by the principal eigenvalue. But for closed manifolds and for the Neumann problem the gap between the principal eigenvalue and the second eigenvalue determines the asymptotic behavior. The understanding of this gap is difficult, since the involved eigenvalues and eigenfunctions are complex-valued. We will give a solution for this problem in this article.

Beyond physical applications there are further reasons to study the enhancement of diffusivity by flows. The growth of computer power has made it possible to solve real-world problems by using so called Markov Chain Monte Carlo Methods (MCMC). See Metropolis and Ulam [24], Hastings [14], Kirkpatrick et al. [19], Brooks [5] for more informations on MCMC. The quality of the MCMC can be described in terms of how fast the involved Markov-process converges toward its equilibrium (see Kushner [21, Marquez [23]). It was proved in Hwang et al. [17. that the addition of a divergence-free drift increases the spectral gap if the first eigenspace of the unperturbed operator is not invariant under the drift. This means that $L_{c}$ with $c=0$ has the smallest spectral gap, and the difference compared with nonzero $c$ is in general strict. This has important implications in the study of MCMC that one should choose the dynamics other than the gradient dynamics (corresponding to $c=0$ ) which is normally used, since the latter has the slowest convergence rate to the underlying probability distribution. In MCMC, general probability distributions $\mu$ (to replace the Lebesgue measure considered here) with energy function $U$ are considered,

$$
\mu(d x)=\frac{1}{Z} \exp (-U(x) / 2) d x,
$$

where $Z$ is the normalizing constant. The distribution can be defined on $\mathbb{R}^{d}$ or a compact subset. Then the operators considered are of the following form:

$$
L_{C}^{U} f(x)=\frac{1}{2} \Delta f(x)+(-\nabla U(x)+C(x)) \nabla f(x),
$$

where the vector field $C$ satisfies

$$
\operatorname{div}(C \exp (-U / 2))=0 .
$$

For each vector field $C$, a diffusion process can be constructed,

$$
d X_{C}(t)=(-\nabla U+C)\left(X_{C}(t)\right) d t+d B(t),
$$


where $B(t)$ is the Brownian motion. The spectral gap of $L_{C}^{U}$ can be used to measure the closeness of the distribution of $X_{C}(t)$ and $\mu$ at large time $t$. Only when $C=$ 0 and $L_{C}^{U}$ is selfadjoint can the spectral gap be calculated approximately by a variational expression. There are huge studies in the literature for the estimate of the spectral gap (sometimes it is also called the first eigenvalue) in this case. See Li and Yau 22, Chen and Wang 7], 8]. See also Chen [6] for the survey and a complete list of references. For $C \neq 0, L_{C}^{U}$ is not selfadjoint. Such a variational expression for the spectral gap is not available for general $C$. Therefore, to calculate the spectral gap numerically is in general very difficult. However, knowing the value of the spectral gap for nonzero $C$ is important for the use of MCMC, since using such a $C$ is preferable because the process has a faster convergence rate to the equilibrium. See Hwang et al. [16], 18, for some calculations. We feel that this research area is still quite open for further study.

1.2. Formal definitions and results. After those considerations we come to the exact formulation of our problem. We fix a closed, compact and connected Riemannian manifold $M$ and a $C^{1}$-vector field $b$ with the property that $\operatorname{div}(b)=0$. On $M$ we have a Riemannian volume, denoted vol. For the integral of a function $f: M \rightarrow \mathbb{R}$ with respect to vol we will use the following abbreviation:

$$
\int f:=\int f(x) \operatorname{vol}(d x) \text {. }
$$

For $f \in C^{2}(M)$ we can define the operator

$$
L_{c} f:=\frac{1}{2} \Delta f+c b \cdot \nabla f .
$$

In the following we will denote by $\left(L_{c}\right.$, Dom $\left.\left(L_{c}\right)\right)$ a closed extension of $\left(L_{c}, C^{2}(M)\right)$. The spectrum of this operator is located in the complex plane and the corresponding eigenfunctions are complex-valued. We therefore introduce the following Hilbert space of mean-zero complex-valued functions:

$$
H:=\left\{\varphi=\varphi_{1}+i \varphi_{2} ; \varphi_{1}, \varphi_{2} \in L^{2}(M): \int \varphi_{1}=\int \varphi_{2}=0\right\}
$$

with scalar product

$$
\begin{aligned}
\langle\varphi, \psi\rangle: & =\int \varphi \bar{\psi} \\
& =\int \varphi_{1} \psi_{1}+\int \varphi_{2} \psi_{2}+i\left(\int \psi_{1} \varphi_{2}-\int \psi_{2} \varphi_{1}\right) .
\end{aligned}
$$

For $\psi=\psi_{1}+i \psi_{2}$ with real $\psi_{1}, \psi_{2}, \bar{\psi}=\psi_{1}-i \psi_{2}$. Therefore,

$$
\langle\varphi, \varphi\rangle=\int|\varphi|^{2} .
$$

We define the following Sobolev space of mean-zero functions:

$$
H^{1}:=\left\{\varphi=\varphi_{1}+i \varphi_{2} \in H ;\left(\left|\nabla \varphi_{1}\right|^{2}+\left|\nabla \varphi_{2}\right|^{2}\right)<\infty\right\}
$$


with scalar product

$$
\begin{aligned}
\langle\varphi, \psi\rangle_{1} & :=\int \nabla \varphi \overline{\nabla \psi} \\
& =\int \nabla \varphi_{1} \cdot \nabla \psi_{1}+\int \nabla \varphi_{2} \cdot \nabla \psi_{2}+i\left(\int \nabla \psi_{1} \cdot \nabla \varphi_{2}-\int \nabla \psi_{2} \cdot \nabla \varphi_{1}\right), \\
\langle\varphi, \varphi\rangle_{1} & =\int|\nabla \varphi|^{2} .
\end{aligned}
$$

The corresponding norms of elements $\varphi \in H$, resp. $\varphi \in H^{1}$, will be denoted by $\|\varphi\|$, resp. $\|\varphi\|_{1}$.

In the following we denote the spectrum of the operator $L_{c}$ by $\operatorname{Spec}\left(L_{c}\right)$. We want to investigate the behavior of the spectral gap

$$
\rho(c):=\inf \left\{\rho ; \exists \mu \in \mathbb{R}:(-\rho+i \mu) \in \operatorname{Spec}\left(L_{c}\right) \backslash\{0\}\right\}
$$

as $c$ goes to infinity. The operator $L_{c}$ generates a semigroup $\left(T_{t}^{(c)}\right)_{t \geq 0}$ of contractions on $L^{2}$.

The RAGE-theorem was used in Constantin et al. to prove the following instead. The statement

The operator $b \cdot \nabla$ has no eigenfunctions in $H^{1}$ is equivalent to the statement:

For all $\delta, \tau>0$ there exists a $c_{o}>0$ such that for all $f \in L^{2}$ and $c \geq c_{o}$ one has

$$
\left\|T_{\tau}^{(c)} f\right\|_{2} \leq \delta\|f\|_{2} .
$$

This last statement implies that $\rho(c)$ diverges to infinity as $c \rightarrow \infty$.

It seems that it rarely happens that the operator $b \cdot \nabla$ has no eigenfunctions in $H^{1}$. In fact, construction of such a $b$ could be difficult. In this paper, we want to understand the behavior of the spectral gap in the situation when the operator $b \cdot \nabla$ has eigenfunctions in $H^{1}$. We define the following eigenspaces:

$$
H_{\mu}^{1}:=\left\{\varphi \in H^{1} ; b \cdot \nabla \varphi \stackrel{w}{=} i \mu \varphi\right\} .
$$

In this article we prove the following result:

Theorem 1. The spectral gap $\rho(c)$ converges to a finite value as $c$ tends to infinity if and only if there exists a $\mu \in \mathbb{R}$ such that $H_{\mu}^{1} \neq \emptyset$. The limit can be expressed in the following way:

$$
\lim _{c \rightarrow \infty} \rho(c)=\inf _{\mu \in \mathbb{R}} \inf \left\{\frac{1}{2} \int|\nabla \varphi|^{2} ; \int|\varphi|^{2}=1, \varphi \in H_{\mu}^{1}\right\} .
$$

Similar results were proved in Berestycki et al. 3 for bounded domains with various boundary conditions. For a bounded measurable potential $V$ and a symmetric matrix-field $A$ satisfying

$$
\sigma_{1}|\xi|^{2} \leq \xi \cdot A \xi \leq \sigma_{2}|\xi|^{2} \text { for all } \xi \in \mathbb{R}^{d} \text { and suitable } \sigma_{1}, \sigma_{2}>0,
$$

they considered the asymptotic behavior of the principal eigenvalue $\lambda(c)$ of the elliptic operators

$$
L_{c} f=\operatorname{div}(A \nabla f)+c b \cdot \nabla f+V f
$$

as $c \rightarrow \infty$ under various boundary conditions including Dirichlet, Neumann and periodic boundary conditions. Their method relies on the fact that the eigenfunction corresponding to the principal eigenvalue is real-valued and positive. Therefore 
they could restrict their considerations to the Hilbert space $H_{0}^{1}$ of real-valued functions, which can be approximated by elements from $C_{c}^{\infty}(\Omega)$ with respect to the first Sobolev norm. They proved the following result:

The sequence $\lambda(c)$ converges iff there exists a weak solution of the equation $b \cdot \nabla \varphi=0$ in $H_{0}^{1}$. If the limit exists, then it can be expressed as

$$
\lim _{c \rightarrow \infty} \lambda(c)=\inf \left\{\int \nabla \varphi \cdot A \nabla \varphi+\int V \varphi^{2} ; \varphi \in H_{0}^{1}, b \cdot \nabla \varphi \stackrel{w}{=} 0, \int \varphi^{2}=1\right\} .
$$

In the discussion section of their paper they raised the question on the behavior of other eigenvalues (see p. 478).

The result of Berestycki et al. 3. motivates the question whether the only eigenvalues that count for establishing the infimum in our result are the first integrals. The following simple examples show that in our situation this is not the case.

Example 1. For $a_{1}>a_{2}$ we can define the following generator:

$$
L_{c} f=\frac{1}{2} \Delta f+c \partial_{x} f
$$

with periodic boundary conditions $f(x, y)=f\left(x+a_{1}, y+a_{2}\right)$.

For all integers $k$ the functions $\varphi_{k}(x, y):=\exp \left(2 \pi k i x / a_{1}\right)$ are eigenfunctions of $\Delta$ for the eigenvalues $-\rho_{k}=-4 \pi^{2} k^{2} / a_{1}^{2}$. Also those functions are eigenfunctions of $c \partial_{x}=c b \cdot \nabla$ corresponding to the nonzero eigenvalue $i c \mu_{k}=i c 2 \pi k / a_{1}$. Therefore it follows that $z_{k}=-\frac{1}{2} \rho_{k}+i c \mu_{k}$ are eigenvalues for the operator $L_{c}$.

On the other hand the kernel of $b \cdot \nabla$ consists of the functions $(x, y) \mapsto \psi(y)$. These functions are generated by $\psi_{k}(x, y):=\exp \left(2 \pi k i y / a_{2}\right)$, where $k$ is an integer. These functions are eigenfunctions of the operator $L_{c}$ corresponding to the eigenvalues $\tilde{z}_{k}=-\tilde{\rho}_{k}=-4 \pi^{2} k^{2} / a_{2}^{2}$. Since $\rho_{1}=4 \pi^{2} / a_{1}^{2}<4 \pi^{2} / a_{2}^{2}=\tilde{\rho}_{1}$ we see that the infimum cannot be reduced to the kernel of $b \cdot \nabla$ in our situation.

Example 2. For two numbers $a_{1}, a_{2} \in \mathbb{R}$ such that $a_{1} / a_{2}$ is irrational we define the following differential operator on $C^{2}\left(\mathbb{R}^{2}\right)$ with periodic boundary conditions of period 1:

$$
L_{c} f=\frac{1}{2} \Delta f+c\left(a_{1} \partial_{x}+a_{2} \partial_{y}\right) f .
$$

The resulting flow is irrational and therefore the kernel has no mean-zero elements in $H^{1}$. However, the functions $f(x, y)=\exp (2 \pi i x)$ and $f(x, y)=\exp (2 \pi i y)$ are $H^{1}$-eigenfunctions of the operator $b \cdot \nabla$ with $b=\left(a_{1}, a_{2}\right)$ with eigenvalues $2 \pi a_{1} i$ and $2 \pi a_{2} i$.

The following argument shows that we can reduce the number of spaces $H_{\mu}^{1}$ in the computation of the limiting spectral gap. Assume that the space $H_{\mu_{0}}^{1}$ contains a nonzero element $\psi$ with $\int|\psi|^{2}=1$. Then we have that

$$
\lim _{c \rightarrow \infty} \rho(c) \leq \frac{1}{2} \int|\nabla \psi|^{2} .
$$

On the other hand we have for all $\mu \in \mathbb{R}$ and $\varphi \in H_{\mu}^{1}$ that

$$
\begin{aligned}
\frac{1}{2} \int|\nabla \varphi|^{2} & \geq \frac{1}{2\|b\|_{\infty}^{2}} \int|b \cdot \nabla \varphi|^{2} \\
& =\frac{\mu^{2}}{2\|b\|_{\infty}^{2}} \int|\varphi|^{2} .
\end{aligned}
$$


Thus it is sufficient to consider the eigenvalues $\mu$ of $b \cdot \nabla$ with

$$
|\mu| \leq\|b\|_{\infty} \sqrt{\int|\nabla \psi|^{2}} .
$$

In the rest of this section, we mention an observation that plays a crucial role for the analysis we use in our study.

1.3. Motivations for the proof. In this subsection we want to show some computations which might help to understand the main idea of the proof of our main theorem, which follows in the next section. Let $\lambda>0$ and consider

$$
L_{c} \psi^{(c)}-\lambda \psi^{(c)}=-g
$$

for real-valued $g \in L^{2}$ satisfying $\int g=0$. This has a unique solution,

$$
\psi^{(c)}(x)=\int_{0}^{\infty} \exp (-\lambda t) T_{t}^{(c)} g(x) d t .
$$

$T^{(c)} g$ is the semigroup generated by $L_{c}$,

$$
\frac{d}{d t} T_{t}^{(c)} g=L_{c} T_{t}^{(c)} g
$$

Since

$$
\begin{aligned}
\frac{d}{d t} \int\left|T_{t}^{(c)} g\right|^{2} & =2 \int T_{t}^{(c)} g L_{c} T_{t}^{(c)} g \\
& =-\int\left|\nabla T^{(c)} g\right|^{2} \\
& \leq-2 \rho(0) \int\left|T_{t}^{(c)} g\right|^{2}
\end{aligned}
$$

we have

$$
\int\left|T_{t}^{(c)} g\right|^{2} \leq \exp (-2 \rho(0) t) \int|g|^{2} .
$$

In the case that $M$ is the torus, we have $\rho(0)=4 \pi^{2}$. Therefore, $\psi^{(c)}$ given above is well defined even for $\lambda=0$ or $\lambda=i c \mu$.

We now consider

$$
L_{c} \psi^{(c)}=i c \mu \psi^{(c)}-g,
$$

where $g=g_{1}+i g_{2} \in H_{\mu}^{1}, H_{\mu}^{1}$ is defined above. We have

$$
b \cdot \nabla g=i \mu g
$$

and

$$
\frac{1}{2} \Delta \psi^{(c)}+c b \cdot \nabla \psi^{(c)}=i c \mu \psi^{(c)}-g .
$$

Multiplying (3) by $\overline{\psi^{(c)}}$ and integrating the relation, we obtain

$$
-\frac{1}{2} \int\left|\nabla \psi^{(c)}\right|^{2}+c \int b \nabla \psi^{(c)} \overline{\psi^{(c)}}=i c \mu \int\left|\psi^{(c)}\right|^{2}-\int g \overline{\psi^{(c)}} .
$$

Since

$$
\int b \nabla \psi^{(c)} \overline{\psi^{(c)}}=-\int \psi^{(c)} b \overline{\nabla \psi^{(c)}}=-\overline{\int b \nabla \psi^{(c)} \overline{\psi^{(c)}}}
$$


this quantity is purely imaginary. Then we have

$$
\frac{1}{2} \int\left|\nabla \psi^{(c)}\right|^{2}=\operatorname{Re}\left(\int g \overline{\psi^{(c)}}\right) .
$$

Again multiplying (3) by $\bar{g}$ and integrating the relation, we obtain

$$
-\frac{1}{2} \int \nabla \psi^{(c)} \overline{\nabla g}+c \int b \nabla \psi^{(c)} \bar{g}=i c \mu \int \psi^{(c)} \bar{g}-\int|g|^{2} .
$$

Using

$$
\int b \nabla \psi^{(c)} \bar{g}=-\int \psi^{(c)} \overline{b \nabla g}=i \mu \int \psi^{(c)} \bar{g}
$$

since $g \in H_{\mu}^{1}$, we obtain

$$
\frac{1}{2} \int \nabla \psi^{(c)} \overline{\nabla g}=\int|g|^{2} .
$$

Here we use the fact that

$$
\int b \cdot \nabla f_{1} f_{2}=-\int b \cdot \nabla f_{2} f_{1}
$$

for all $f_{1}, f_{2}$.

By applying the Hölder inequality to (5), (6), we can show

$$
\left(\frac{1}{2} \int\left|\nabla \psi^{(c)}\right|^{2}\right)^{2} \leq\left(\int|g|^{2}\right)\left(\int\left|\psi^{(c)}\right|^{2}\right)
$$

and

$$
\left(\int|g|^{2}\right)^{2} \leq\left(\frac{1}{2} \int\left|\nabla \psi^{(c)}\right|^{2}\right)\left(\frac{1}{2} \int|\nabla g|^{2}\right) .
$$

From these two relations, we have

$$
\frac{\frac{1}{2} \int\left|\nabla \psi^{(c)}\right|^{2}}{\int\left|\psi^{(c)}\right|^{2}} \leq \frac{\frac{1}{2} \int|\nabla g|^{2}}{\int|g|^{2}}
$$

This suggests that if $g=g_{1}+i g_{2} \in H_{\mu}^{1}$ attains the minimum of the following,

$$
\rho_{\mu}=\inf \left\{\frac{\frac{1}{2} \int|\nabla \psi|^{2}}{\int|\psi|^{2}} ; \psi=\psi_{1}+i \psi_{2} \neq 0 \in H_{\mu}^{1}\right\}
$$

then any limit of $\psi^{(c)}$ is also an element of $H_{\mu}^{1}$ and attains the minimum (see $\rho_{\mu}$ also in Section 2).

Let $\psi^{*}=\psi_{1}^{*}+i \psi_{2}^{*}$ denote a limit of $\psi^{(c)}$. Assume the uniqueness of $g$ taking maximum in $\rho_{\mu}$ (up to the multiplication of constants). Then

$$
\psi^{*}=k g
$$

for some constant $k$. The above calculations show that we must have

$$
\frac{1}{k}=\rho_{\mu} .
$$

Therefore, we have the picture

$$
L_{c} \psi^{(c)}=i c \mu \psi^{(c)}-g \sim\left(-\rho_{\mu}+i c \mu\right) \psi^{(c)} .
$$

This suggests that an eigenvalue of $L_{c}$ close to $-\rho_{\mu}+i c \mu$ can be found (Theorem 2 stated in Section 2 gives the precise statement). 
Here is the organization of the paper. Section 2 gives the main results and proofs. In Section 3, we present some examples; some are from geometry, and some are from the consideration of MCMC.

\section{THE MAIN RESULTS AND PROOFS}

Proposition 1. The operators $L_{c}$ have no continuous spectrum. For all $c>0$, $z \in \operatorname{Spec}\left(L_{c}\right)$ if and only if $z$ is an eigenvalue of $L_{c}$ or $\bar{z}$ is an eigenvalue of $L_{-c}$.

Proof. Assume that $z:=\rho+i \mu \in \operatorname{Spec}\left(L_{c}\right)$ is not an eigenvalue of $L_{c}$. Since $z \in \operatorname{Spec}\left(L_{c}\right)$, we have that $\left(L_{c}-z\right)$ has no bounded inverse with dense domain of definition. But since $z$ is not an eigenvalue, this means that $\left(L_{c}-z\right)$ is one-to-one. Then either $\left(L_{c}-z\right)^{-1}$ is not densely defined (residual spectrum) or $\left(L_{c}-z\right)^{-1}$ is densely defined but unbounded (continuous spectrum).

We first prove that $z$ is not in the continuous spectrum of $L_{c}$. For this it is sufficient to prove that the range of $\left(L_{c}-z\right)$ is closed in $H$. This follows from the following consideration. Assume $z$ is in the continuous spectrum of $L_{c}$. Then the range of $L_{c}-z$ is dense. Therefore, if we know that the range of $\left(L_{c}-z\right)$ is closed and dense, then it follows that $\left(L_{c}-z\right)^{-1}$ is defined everywhere. By the closed graph theorem this would imply that $\left(L_{c}-z\right)^{-1}$ is bounded and defined everywhere, i.e., that $z$ is not in $\operatorname{Spec}\left(L_{c}\right)$, a contradiction.

We now prove that $\left(L_{c}-z\right)$ has closed range. Assume that there exists a sequence $f_{n} \in H^{1}$ and a $g \in H$ such that $\left(L_{c}-z\right) f_{n} \rightarrow g$ in $H$. Then it follows that

$$
-\frac{1}{2}\left(\left\langle\left(L_{c}-z\right) f_{n}, f_{n}\right\rangle+\left\langle f_{n},\left(L_{c}-z\right) f_{n}\right\rangle\right)=\left\|f_{n}\right\|_{1}^{2}+\rho\left\|f_{n}\right\|^{2} .
$$

Application of the Cauchy-Schwarz inequality to the left side of this equation yields

$$
\left|\left\|f_{n}\right\|_{1}^{2}+\rho\left\|f_{n}\right\|^{2}\right| \leq\left\|\left(L_{c}-z\right) f_{n}\right\|\left\|f_{n}\right\| \leq(1+\|g\|)\left\|f_{n}\right\| \text { for large } n \in \mathbb{N} \text {. }
$$

First assume that the sequence $\left\|f_{n}\right\|$ is unbounded. We then can assume without loss of generality that $\left\|f_{n}\right\| \uparrow \infty$ as $n \rightarrow \infty$. Then the sequence $\hat{f}_{n}:=f_{n} /\left\|f_{n}\right\|$ satisfies $\left(L_{c}-z\right) \hat{f}_{n} \rightarrow 0$ in $H$ as $n \rightarrow \infty$. The same reasoning as above now implies

$$
\left|\left\|\hat{f}_{n}\right\|_{1}^{2}+\rho\right| \leq\left\|\left(L_{c}-z\right) \hat{f}_{n}\right\|\left\|\hat{f}_{n}\right\| \longrightarrow 0
$$

This implies that $\hat{f}_{n}$ is bounded in $H^{1}$. Rellich's lemma then implies that there exists a subsequence $\hat{f}_{n_{k}}, k \in \mathbb{N}$ and an element $\hat{\psi} \in H^{1}$ such that $\hat{f}_{n_{k}} \rightarrow \hat{\psi}$ in $H$ and weakly in $H^{1}$. It then follows from the fact that $L_{c}$ is closed that $\hat{\psi} \in \operatorname{Dom}\left(L_{c}\right)$ and that $\left(L_{c}-z\right) \hat{\psi}=0$. This is a contradiction, since then $\hat{\psi}$ is an eigenfunction of $L_{c}$ corresponding to the eigenvalue $z$; i.e., $z$ is not in the continuous spectrum of $L_{c}$.

Therefore, the sequence $\left\|f_{n}\right\|$ must be bounded. But this then implies that $\left\|f_{n}\right\|_{1}$ is bounded. By Rellich's lemma there exists a subsequence $f_{n_{k}}, k \in \mathbb{N}$ and a $\psi \in H^{1}$ such that $f_{n_{k}}$ converges toward $\psi$ in $H$ and weakly in $H^{1}$. Since the operator $L_{c}$ is closed, this implies that $\left(L_{c}-z\right) \psi=g$, i.e. that $g$ is in the range of $\left(L_{c}-z\right)$.

We can conclude that the range of $L_{c}-z$ is not dense. This implies that there exists a $\varphi \in \overline{\operatorname{Ran}\left(L_{c}-z\right)} \perp$. Then for all $\psi \in \operatorname{Dom}\left(L_{c}\right)$ we have

$$
0=\left\langle\varphi,\left(L_{c}-z\right) \psi\right\rangle=\left\langle\left(L_{c}^{*}-\bar{z}\right) \varphi, \psi\right\rangle .
$$

This means that $\varphi$ is in $\operatorname{Ker}\left(L_{c}^{*}-\bar{z}\right)$, i.e. that $\varphi$ is an eigenfunction of $L_{c}^{*}=L_{-c}$ corresponding to the eigenvalue $\bar{z}=\rho-i \mu$. 
For $\mu \in \mathbb{R}$ we define the following subspace:

$$
H_{\mu}^{1}:=\left\{\varphi \in H^{1} ; b \cdot \nabla \varphi \stackrel{w}{=} i \mu \varphi\right\}
$$

We first need the following lemma on partial integration.

Lemma 1. For $\varphi \in H^{1}$ we have

$$
\int \varphi b \cdot \nabla \varphi=0
$$

Proof. We found the following proof in Berestycki et al. [3] (p. 455):

Since $\varphi \in H^{1}$ it follows that $\varphi^{2} \in W^{1,1}$. Therefore, $\varphi^{2}$ can be approximated in $W^{1,1}$ by a sequence $\chi_{n}$ from $C^{\infty}$. It then follows from $\operatorname{div}(b)=0$ that

$$
\int \varphi b \cdot \nabla \varphi=\frac{1}{2} \int b \cdot \nabla\left(\varphi^{2}\right)=\lim _{n \rightarrow \infty} \frac{1}{2} \int b \cdot \nabla \chi_{n}=0 .
$$

Proposition 2. If $\liminf _{c \rightarrow \infty} \rho(c)<\infty$, then it follows that

$$
\liminf _{c \rightarrow \infty} \rho(c) \geq \inf \left\{\frac{1}{2} \int|\nabla \varphi|^{2} ; \int|\varphi|^{2}=1, \exists \mu \in \mathbb{R}: \varphi \in H_{\mu}^{1}\right\} .
$$

Proof. Let $\varphi^{(c)}=\varphi_{1}^{(c)}+i \varphi_{2}^{(c)} \in H^{1}$ be a solution of $L_{c} \varphi^{(c)}=\left(-\rho(c)+i \mu_{c}\right) \varphi^{(c)}$ with

That is,

$$
\int\left|\varphi^{(c)}\right|^{2}=1
$$

$$
\frac{1}{2} \Delta \varphi^{(c)}+c b \nabla \varphi^{(c)}=\left(-\rho(c)+i \mu_{c}\right) \varphi^{(c)} .
$$

Multiplication of (7) with $\overline{\varphi^{(c)}}$, integration and application of Lemma 1 yields

$$
\begin{aligned}
& -\frac{1}{2} \int\left|\nabla \varphi^{(c)}\right|^{2}+c \int b \cdot \nabla \varphi^{(c)} \overline{\varphi^{(c)}} \\
= & \left(-\rho(c)+i \mu_{c}\right) \int\left|\varphi^{(c)}\right|^{2} .
\end{aligned}
$$

As in (4), $\int b \cdot \nabla \varphi^{(c)} \overline{\varphi^{(c)}}$ is purely imaginary. Analyzing the real part of (8) then implies that

$$
\frac{1}{2} \int\left|\nabla \varphi^{(c)}\right|^{2}=\rho(c)
$$

The assumption $\liminf _{c \rightarrow \infty} \rho(c)<\infty$ together with Rellich's theorem yields that there exists a subsequence $\varphi^{\left(c_{n}\right)}$ of $\varphi^{(c)}$ with $c_{n} \uparrow \infty$ and an element $\varphi \in H^{1}$ such that $\varphi^{\left(c_{n}\right)}$ converges toward $\varphi$ as $n \rightarrow \infty$ in $L^{2}$ and weakly in $H^{1}$.

Now, take an arbitrary smooth real function $\psi$ defined on $M$. We multiply (7) by $\psi$, integrate and divide by $c_{n}>0$ to obtain

$$
-\frac{1}{2 c_{n}} \int \nabla \varphi^{\left(c_{n}\right)} \cdot \nabla \psi-\int \varphi^{\left(c_{n}\right)} b \cdot \nabla \psi=\frac{-\rho\left(c_{n}\right)+i \mu_{c_{n}}}{c_{n}} \int \varphi^{\left(c_{n}\right)} \psi .
$$

Since as $n \rightarrow \infty$,

$$
\left|\int \nabla \varphi^{\left(c_{n}\right)} \cdot \nabla \psi\right|^{2} \leq \int\left|\nabla \varphi^{\left(c_{n}\right)}\right|^{2} \int|\nabla \psi|^{2} \text { and }\left|\int \varphi^{\left(c_{n}\right)} \psi\right|^{2} \leq \int \psi^{2}
$$


are bounded, taking the limit as $n \rightarrow \infty$ yields

$$
-\int \varphi b \cdot \nabla \psi=\lim _{n \rightarrow \infty} \frac{i \mu_{c_{n}}}{c_{n}} \int \varphi \psi
$$

In particular, $\mu_{c_{n}} / c_{n}$ converges toward a $\mu \in \mathbb{R}$ such that $b \cdot \nabla \varphi \stackrel{w}{=} i \mu \varphi$. It then follows that

$$
\lim _{n \rightarrow \infty} \rho\left(c_{n}\right)=\lim _{n \rightarrow \infty} \frac{1}{2} \int\left|\nabla \varphi^{\left(c_{n}\right)}\right|^{2} \geq \frac{1}{2} \int|\nabla \varphi|^{2} .
$$

Since the same reasoning holds for every subsequence, the result follows.

For the following we will need

$$
M_{\mu}:=\left\{\psi \in H_{\mu}^{1} ; \frac{1}{2} \int|\nabla \psi|^{2}=\rho_{\mu} \int|\psi|^{2}\right\},
$$

where

$$
\rho_{\mu}:=\inf \left\{\frac{1}{2} \int|\nabla \psi|^{2} ; \psi \in H_{\mu}^{1}, \int|\psi|^{2}=1\right\} .
$$

We will see, as a consequence of Lemma 4 given later, that $M_{\mu}$ contains a nonzero element if $H_{\mu}^{1}$ contains a nonzero element. The following two lemmas for the spaces $M_{\mu}$ will be useful in our analysis:

Lemma 2. Assume that $H_{\mu}^{1} \neq\{0\}$ and $\varphi \in H_{\mu}^{1}$. Then $\varphi \in M_{\mu}$ if and only if for all $\psi \in H_{\mu}^{1}$ one has

$$
\frac{1}{2} \int \nabla \varphi \cdot \overline{\nabla \psi}=\rho_{\mu} \int \varphi \bar{\psi}
$$

Proof. If the relation (9) holds for all $\psi \in H_{\mu}^{1}$, then it holds in particular for $\varphi$. This implies that $\varphi$ is in $M_{\mu}$.

On the other hand, if $\varphi=\varphi_{1}+i \varphi_{2}$ is in $M_{\mu}$ and $\psi=\psi_{1}+i \psi_{2}$ is in $H_{\mu}^{1}$, then for all $t \in \mathbb{R}$ the functions $\psi(t):=\varphi+t \psi$ are in $H_{\mu}^{1}$. Denote $\psi(t)=\psi_{1}(t)+i \psi_{2}(t)$. The function

$$
t \mapsto \frac{1}{2} \int\left(\left|\nabla \psi_{1}(t)\right|^{2}+\left|\nabla \psi_{2}(t)\right|^{2}\right) / \int\left(\left(\psi_{1}(t)\right)^{2}+\left(\psi_{2}(t)\right)^{2}\right)
$$

is differentiable and minimal at zero. Differentiation at zero yields

$$
\begin{aligned}
0= & \frac{1}{2} \int\left(\nabla \varphi_{1} \nabla \psi_{1}+\nabla \varphi_{2} \nabla \psi_{2}\right) / \int\left(\varphi_{1}^{2}+\varphi_{2}^{2}\right) \\
& -\frac{1}{2} \int\left(\varphi_{1} \psi_{1}+\varphi_{2} \psi_{2}\right) \int\left(\left|\nabla \varphi_{1}\right|^{2}+\left|\nabla \varphi_{2}\right|^{2}\right) /\left(\int\left(\varphi_{1}^{2}+\varphi_{2}^{2}\right)\right)^{2} .
\end{aligned}
$$

Together with the definition of $\rho_{\mu}$ this yields

$$
\frac{1}{2} \int\left(\nabla \varphi_{1} \nabla \psi_{1}+\nabla \varphi_{2} \nabla \psi_{2}\right)=\rho_{\mu} \int\left(\varphi_{1} \psi_{1}+\varphi_{2} \psi_{2}\right) .
$$

Since $i \psi$ is also in $H_{\mu}^{1}$, the above argument can be applied to $i \psi$ to get

$$
\frac{1}{2} \int\left(-\nabla \varphi_{1} \nabla \psi_{2}+\nabla \varphi_{2} \nabla \psi_{1}\right)=\rho_{\mu} \int\left(-\varphi_{1} \psi_{2}+\varphi_{2} \psi_{1}\right) .
$$

The result follows from these two relations.

Lemma 3. The set $M_{\mu}$ is a finite-dimensional $\mathbb{C}$-vector-space. 
Proof. The vector-space property follows from the previous lemma. To prove that the dimension of $M_{\mu}$ is finite we assume that this is not the case. Then we can extract an orthonormal sequence $\psi^{(n)}$ such that

$$
\frac{1}{2} \int\left|\nabla \psi^{(n)}\right|^{2}=\rho_{\mu} .
$$

It then follows from Rellich's theorem that $\psi^{(n)}$ has $L^{2}$-convergent subsequences. This is however not possible since $\psi^{(n)}$ is orthonormal.

Lemma 4. There exists a $\delta>0$ such that for all $\psi=\psi_{1}+i \psi_{2} \in H_{\mu}^{1} \backslash\{0\}$,

$$
\int \psi \bar{\varphi}=0 \quad \text { for all } \varphi \in M_{\mu}
$$

implies

$$
\frac{1}{2} \int|\nabla \psi|^{2} \geq\left(\rho_{\mu}+\delta\right) \int|\psi|^{2} .
$$

Proof. Assume that for every $n \in \mathbb{N}$ there exists a $\psi^{(n)} \in H_{\mu}^{1} \backslash\{0\}$ such that

$$
\int \psi^{(n)} \bar{\varphi}=0 \quad \text { for all } \varphi \in M_{\mu}
$$

and

$$
\frac{1}{2} \int\left|\nabla \psi^{(n)}\right|^{2} \leq\left(\rho_{\mu}+\frac{1}{n}\right) \int\left|\psi^{(n)}\right|^{2} .
$$

Without loss of generality we can assume that

$$
\int\left|\psi^{(n)}\right|^{2}=1 .
$$

Since $\psi^{(n)}$ cannot be in $M_{\mu}$, there exists a $\left.\delta_{n} \in\right] 0,1 / n[$ such that

$$
\frac{1}{2} \int\left|\nabla \psi^{(n)}\right|^{2}=\left(\rho_{\mu}+\delta_{n}\right) .
$$

It now follows from Rellich's lemma that $\left(\psi^{(n)}\right)$ has $L^{2}$-convergent and weakly $H^{1}$ convergent subsequences (see Richtmyer [26, p.115). The limit-point $\psi^{*}$ satisfies

$$
\frac{1}{2} \int\left|\nabla \psi^{*}\right|^{2} \leq \rho_{\mu} \int\left|\psi^{*}\right|^{2}
$$

and is thus a member of $M_{\mu}$. Furthermore, it follows from (10) that

$$
\int \psi^{*} \bar{\varphi}=0 \text { for all } \varphi \in M_{\mu} .
$$

These two facts imply that $\psi^{*}=0$. This is however a contradiction, since it follows from (11) that

$$
\int\left|\psi^{*}\right|^{2}=1 .
$$

Theorem 2. Assume that $H_{\mu}^{1} \neq\{0\}$. Then for all $\alpha>0$ there exists a $c_{o}$ such that for all $c \geq c_{o}$ there exists a $\tilde{z}=-\tilde{\rho}+i \tilde{\mu} \in B_{\alpha}\left(-\rho_{\mu}+i c \mu\right)$ such that $\tilde{z} \in \operatorname{Spec}\left(L_{c}\right)$. 2 .

We now prove Theorem 1 in the introduction from Theorem 2 and Proposition 
Proof of Theorem 1. If the limit exists, then $\lim \sup \rho(c)$ is bounded and it follows from Proposition 2 that

$$
\liminf _{c \rightarrow \infty} \rho(c) \geq \inf \left\{\frac{1}{2} \int|\nabla \varphi|^{2} ; \int|\varphi|^{2}=1, \exists \mu \in \mathbb{R}: \varphi \in H_{\mu}^{1}\right\} .
$$

In particular one of the spaces $H_{\mu}^{1}$ must contain a nonzero element.

If $\mu \in \mathbb{R}$ is such that $H_{\mu}^{1}$ has a nonzero element, then we have from Theorem 2 that

$$
\limsup _{c \rightarrow \infty} \rho(c) \leq \inf \left\{\frac{1}{2} \int|\nabla \varphi|^{2} ; \int|\varphi|^{2}=1, \varphi \in H_{\mu}^{1}\right\} .
$$

Together, we obtain

$$
\lim _{c \rightarrow \infty} \rho(c)=\inf \left\{\frac{1}{2} \int|\nabla \varphi|^{2} ; \int|\varphi|^{2}=1, \exists \mu \in \mathbb{R}: \varphi \in H_{\mu}^{1}\right\} .
$$

For the proof of Theorem 2, we proceed by contradiction. Assume $H_{\mu}^{1}$ has nonzero elements and that there exist $\alpha>0, c_{n} \rightarrow \infty$ such that for any element

$$
(-\tilde{\rho}, \tilde{\mu}) \in\left\{\left(-\rho^{\prime}, \mu^{\prime}\right):\left|\rho^{\prime}-\rho_{\mu}\right|^{2}+\left|\mu^{\prime}-c_{n} \mu\right|^{2} \leq \alpha^{2}\right\}
$$

we have that $-\tilde{\rho}+i \tilde{\mu}$ is not in the spectrum of $L_{c_{n}}$, i.e. that $\left(L_{c_{n}}-(-\tilde{\rho}+i \tilde{\mu})\right)^{-1}$ exists and is bounded.

Fix $g=g_{1}+i g_{2} \in M_{\mu}$ with the property

$$
\int|g|^{2}=1
$$

Take any pair $\epsilon, \delta>0$ with $\epsilon^{2}+\delta^{2} \leq \alpha^{2}$ such that $\tilde{\rho}=\rho_{\mu}+\epsilon$ and $\tilde{\mu}=c_{n} \mu+\delta$.

Since $-\tilde{\rho}+i \tilde{\mu}$ is not in the spectrum of $L_{c_{n}}$, there exists a $\varphi^{\left(c_{n}\right)} \in H^{1}$ such that

$$
L_{c_{n}} \varphi^{\left(c_{n}\right)}=(-\tilde{\rho}+i \tilde{\mu}) \varphi^{\left(c_{n}\right)}-g .
$$

In the following we use $\varphi_{z}^{\left(c_{n}\right)}$ for $\varphi^{\left(c_{n}\right)}$ if we want to emphasize the dependence on $z=\epsilon+i \delta$. The following lemmas hold.

Lemma 5. The sequence $\left(\varphi^{\left(c_{n}\right)}\right)$ is either unbounded in $L^{2}$ or there exist a $\varphi^{*} \in H^{1}$ and a subsequence of $\left(\varphi^{\left(c_{n}\right)}\right)$, which converges toward $\varphi^{*}$ in $L^{2}$ and weakly in $H^{1}$.

Proof. Assume that the sequence is bounded in $L^{2}$. We use the equation for $\varphi^{\left(c_{n}\right)}$. In order to avoid cumbersome notation we will write $c$ for $c_{n}$, and $\varphi$ for $\varphi^{(c)}$. We have the equation

$$
\frac{1}{2} \Delta \varphi+c b \cdot \nabla \varphi=(-\tilde{\rho}+i \tilde{\mu}) \varphi-g .
$$

Multiplication of (13) by $\bar{\varphi}$ and doing an integration of the relation yields

$$
-\frac{1}{2} \int|\nabla \varphi|^{2}+c \int b \cdot \nabla \varphi \bar{\varphi}=(-\tilde{\rho}+i \tilde{\mu}) \int|\varphi|^{2}-\int g \bar{\varphi} .
$$

Since $\int b \cdot \nabla \varphi \bar{\varphi}$ is purely imaginary (see (4)), by taking the real part in (14) we have

$$
\frac{1}{2} \int|\nabla \varphi|^{2}=\tilde{\rho} \int|\varphi|^{2}+\operatorname{Re}\left(\int g \bar{\varphi}\right)
$$


Thus an $L^{2}$-bound leads to an $H^{1}$-bound. Then it follows from Rellich's theorem that there exists a subsequence of $\left(\varphi^{\left(c_{n}\right)}\right)$ which converges in $L^{2}$ and weakly in $H^{1}$ toward a suitable $\varphi^{*} \in H^{1}$.

We first investigate the case when $\left(\varphi^{\left(c_{n}\right)}\right)$ is bounded in $L^{2}$, i.e. when there exists a $\varphi^{*} \in H^{1}$ and a subsequence of $\varphi^{\left(c_{n}\right)}$ which converges toward $\varphi^{*}$ in $L^{2}$. Without

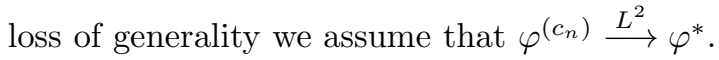

We will prove the following statement.

Lemma 6. If $\left(\varphi^{\left(c_{n}\right)}\right)$ converges toward $\varphi^{*}$ in $L^{2}$ and weakly in $H^{1}$, then $\varphi^{*} \in H_{\mu}^{1}$.

Proof. Multiplication of equations (13) with an arbitrary real $\psi \in H^{1}$ gives after integration and division by $c$ that

$$
-\frac{1}{2 c_{n}} \int \nabla \psi \nabla \varphi^{\left(c_{n}\right)}+\int b \cdot \nabla \varphi^{\left(c_{n}\right)} \psi=-\frac{\tilde{\rho}+i \tilde{\mu}}{c_{n}} \int \psi \varphi^{\left(c_{n}\right)}-\frac{1}{c_{n}} \int \psi g .
$$

Since $\tilde{\mu} / c_{n} \rightarrow \mu$ as $c_{n} \rightarrow \infty$ we obtain

$$
-\int \varphi^{*} b \cdot \nabla \psi=-i \mu \int \varphi^{*} \psi
$$

The result follows.

Lemma 7. If $\left(\varphi^{\left(c_{n}\right)}\right)$ converges toward $\varphi^{*}$ in $L^{2}$ and weakly in $H^{1}$, then one has

$$
(\epsilon-i \delta) \int \varphi^{*} \bar{g}=-1
$$

Proof. Now we multiply (13) by $\bar{g}$ to obtain after integration

$$
-\frac{1}{2} \int \nabla \varphi \overline{\nabla g}+c \int b \cdot \nabla \varphi \bar{g}=(-\tilde{\rho}+i \tilde{\mu}) \int \varphi \bar{g}-\int|g|^{2} .
$$

By $b \nabla g=i \mu g$, we have

$$
\int b \cdot \nabla \varphi \bar{g}=-\int \varphi b \cdot \nabla \bar{g}=i \mu \int \varphi \bar{g} .
$$

Then using $\tilde{\mu}=c \mu+\delta$, (16) becomes

$$
-\frac{1}{2} \int \nabla \varphi \overline{\nabla g}=(-\tilde{\rho}+i \delta) \int \varphi \bar{g}-\int|g|^{2} .
$$

Taking the limit $c \rightarrow \infty$, we obtain

$$
\frac{1}{2} \int \nabla \varphi^{*} \overline{\nabla g}=(\tilde{\rho}-i \delta) \int \varphi^{*} \bar{g}+\int|g|^{2} .
$$

Since $g \in M_{\mu}$, by Lemma 2 and Lemma 6, we have

$$
\frac{1}{2} \int \nabla \varphi^{*} \overline{\nabla g}=\rho_{\mu} \int \varphi^{*} \bar{g} .
$$

Together we have

$$
(\epsilon-i \delta) \int \varphi^{*} \bar{g}=-\int|g|^{2}=-1
$$


Lemma 8. Assume $\left(\varphi^{\left(c_{n}\right)}\right)$ converges toward $\varphi^{*}$ in $L^{2}$ and weakly in $H^{1}$. Then if $f \in M_{\mu}$ and

$$
\int g \bar{f}=0
$$

we have

$$
\int \varphi^{*} \bar{f}=0
$$

Proof. We multiply (13) by $\bar{f}$ and integrate to obtain the formulas

$$
-\frac{1}{2} \int \nabla \varphi \overline{\nabla f}+c \int b \cdot \nabla \varphi \bar{f}=(-\tilde{\rho}+i \tilde{\mu}) \int \varphi \bar{f} .
$$

The property $f \in M_{\mu}$ implies that $b \nabla f=i \mu f$. Then

$$
\int b \cdot \nabla \varphi \bar{f}=-\int \varphi b \cdot \nabla \bar{f}=i \mu \int \varphi \bar{f},
$$

and (17) becomes

$$
-\frac{1}{2} \int \nabla \varphi \overline{\nabla f}=(-\tilde{\rho}+i \delta) \int \varphi \bar{f}
$$

Taking $c \rightarrow \infty$,

$$
-\frac{1}{2} \int \nabla \varphi^{*} \overline{\nabla f}=(-\tilde{\rho}+i \delta) \int \varphi^{*} \bar{f} .
$$

Since $f \in M_{\mu}$, by Lemma 2 ,

$$
\frac{1}{2} \int \nabla f \overline{\nabla \varphi^{*}}=\rho_{\mu} \int f \overline{\varphi^{*}}
$$

That is,

$$
\frac{1}{2} \int \nabla \varphi^{*} \overline{\nabla f}=\rho_{\mu} \int \varphi^{*} \bar{f}
$$

Then (18) becomes

$$
(\epsilon-i \delta) \int \varphi^{*} \bar{f}=0
$$

This completes the proof.

Lemma 9. If $\left(\varphi^{\left(c_{n}\right)}\right)$ converges toward $\varphi^{*}$ in $L^{2}$ and weakly in $H^{1}$, then

$$
\hat{\varphi}^{*}:=\varphi^{*}+\frac{\epsilon+i \delta}{\epsilon^{2}+\delta^{2}} g
$$

satisfies the properties that $\hat{\varphi}^{*} \in H_{\mu}^{1}$ and

$$
\frac{1}{2} \int\left|\nabla \hat{\varphi}^{*}\right|^{2} \leq \tilde{\rho} \int\left|\hat{\varphi}^{*}\right|^{2}
$$

Proof. In (15), we take $c \rightarrow \infty$ to obtain

$$
\begin{aligned}
\frac{1}{2} \int\left|\nabla \varphi^{*}\right|^{2} & \leq \tilde{\rho} \int\left|\varphi^{*}\right|^{2}+\operatorname{Re}\left(\int g \overline{\varphi^{*}}\right) \\
& =\tilde{\rho} \int\left|\varphi^{*}\right|^{2}-\frac{\epsilon}{\epsilon^{2}+\delta^{2}} .
\end{aligned}
$$


Here we use Lemma 7. Then

$$
\begin{aligned}
\frac{1}{2} \int\left|\nabla \hat{\varphi}^{*}\right|^{2}= & \frac{1}{2} \int\left|\nabla \varphi^{*}\right|^{2}+\frac{1}{2} \int|\nabla g|^{2} \frac{1}{\epsilon^{2}+\delta^{2}} \\
& +\frac{1}{2} \int \nabla \varphi^{*} \overline{\nabla g} \frac{\epsilon-i \delta}{\epsilon^{2}+\delta^{2}}+\frac{1}{2} \int \overline{\nabla \varphi^{*}} \nabla g \frac{\epsilon+i \delta}{\epsilon^{2}+\delta^{2}} .
\end{aligned}
$$

Using

$$
\begin{gathered}
\frac{1}{2} \int|\nabla g|^{2}=\rho_{\mu} \int|g|^{2}=\rho_{\mu}, \\
\frac{1}{2} \int \nabla \varphi^{*} \overline{\nabla g}=\rho_{\mu} \int \varphi^{*} \bar{g}=-\rho_{\mu} \frac{\epsilon+i \delta}{\epsilon^{2}+\delta^{2}},
\end{gathered}
$$

we have

$$
\frac{1}{2} \int\left|\nabla \hat{\varphi}^{*}\right|^{2}=\frac{1}{2} \int\left|\nabla \varphi^{*}\right|^{2}-\rho_{\mu} \frac{1}{\epsilon^{2}+\delta^{2}}
$$

We also have

$$
\operatorname{Re}\left(\int g \overline{\varphi^{*}}\right)=-\frac{\epsilon}{\epsilon^{2}+\delta^{2}} .
$$

From these relations and (19), we have

$$
\frac{1}{2} \int\left|\nabla \hat{\varphi}^{*}\right|^{2} \leq \tilde{\rho} \int\left|\varphi^{*}\right|^{2}-\tilde{\rho} \frac{1}{\epsilon^{2}+\delta^{2}}=\tilde{\rho} \int\left|\hat{\varphi}^{*}\right|^{2} .
$$

This completes the proof.

Lemma 10. There exists an $\epsilon_{o}>0$ such that for all $\left.\epsilon \in\right]-\epsilon_{o}, \epsilon_{o}[$, one has that if $\left(\varphi^{\left(c_{n}\right)}\right)$ converges toward $\varphi^{*}$ in $L^{2}$ and weakly in $H^{1}$, then it follows that

$$
\varphi^{*}=-\frac{\epsilon+i \delta}{\epsilon^{2}+\delta^{2}} g .
$$

Proof. We define

$$
\hat{\varphi}^{*}:=\varphi^{*}+\frac{\epsilon+i \delta}{\epsilon^{2}+\delta^{2}} g
$$

We need to prove $\hat{\varphi}^{*}=0$.

First, let $h \in M_{\mu}$ satisfy $h \perp g$. Then we have that

$$
\int h \bar{g}=0
$$

Now, we use Lemma 8 with $f=h$ to obtain

$$
\int \varphi^{*} \bar{h}=0 \text {. }
$$

This then implies that $\varphi^{*} \perp h$. Together with the assumption $g \perp h$, we obtain $\hat{\varphi}^{*} \perp h$.

Now, we take $h=g$. From Lemma 7 ,

$$
\int \varphi^{*} \bar{g}=-\frac{\epsilon+i \delta}{\epsilon^{2}+\delta^{2}}
$$

It then follows that $\hat{\varphi}^{*} \perp g$. Since with respect to $g$ we can always decompose a general $h \in M_{\mu}$ as $h=h^{\perp}+\kappa g$ with $h^{\perp} \perp g$ and a suitable $\kappa$, we have $\hat{\varphi}^{*} \perp M_{\mu}$. 
This implies that $\hat{\varphi}^{*}=0$; otherwise, by Lemma 4 there is $\delta_{0}>0$ such that

$$
\frac{1}{2} \int\left|\nabla \hat{\varphi}^{*}\right|^{2} \geq\left(\rho_{\mu}+\delta_{0}\right) \int\left|\hat{\varphi}^{*}\right|^{2},
$$

but this contradicts with Lemma 9 if $\epsilon_{0}$ (and hence $\delta$ ) is small enough.

Lemma 11. We have for all $\alpha>0$ small enough that

$$
\limsup _{c \rightarrow \infty} \sup _{|\epsilon|^{2}+|\delta|^{2}=\alpha} \int\left|\varphi_{\epsilon+i \delta}^{(c)}\right|^{2}<\infty .
$$

Proof. We assume that there exists a sequence $\tilde{\rho}_{n}=\rho_{\mu}+\epsilon_{n}, \tilde{\mu}_{n}=c_{n} \mu+\delta_{n}$ and $c_{n}>0$ such that $\left|\epsilon_{n}\right|^{2}+\left|\delta_{n}\right|^{2}=\alpha, z_{n}=\epsilon_{n}+i \delta_{n}, c_{n} \rightarrow \infty$ and

$$
K_{n}:=\int\left|\varphi_{z_{n}}^{\left(c_{n}\right)}\right|^{2} \rightarrow \infty
$$

The sequence $\psi^{(n)}:=\varphi^{(n)} / K_{n}$ is bounded in $L^{2}$ and satisfies the equations

$$
\frac{1}{2} \Delta \psi^{(n)}+c_{n} b \cdot \nabla \psi^{(n)}=\left(-\tilde{\rho}_{n}+i \mu_{n}\right) \psi^{(n)}-g / K_{n} .
$$

It follows by the same argument as in the proof of Lemma 5 that the sequence $\left(\psi^{(n)}\right)$ is also bounded in $H^{1}$. Thus there exist a $\psi^{*} \in H^{1}$ and a subsequence from $\left(\psi^{(n)}\right)$ which converges toward $\psi^{*}$ in $L^{2}$ and weakly in $H^{1}$. Furthermore the sequences $\epsilon_{n}$ and $\delta_{n}$ are bounded, and therefore we can assume without loss of generality that they converge toward suitable $\epsilon, \delta$ such that $\epsilon^{2}+\delta^{2}=\alpha$. In the following we will write $\psi$ for $\psi^{(n)}$ in order to avoid overloaded notation.

The following argument is almost identical to the arguments for Lemma 7 and Lemma 8 . We sketch the main steps.

We multiply (20) by $\overline{\psi^{(n)}}$ and integrate to obtain

$$
-\frac{1}{2} \int\left|\nabla \psi^{(n)}\right|^{2}+c_{n} \int b \nabla \psi^{(n)} \overline{\psi^{(n)}}=\left(-\tilde{\rho}_{n}+i \tilde{\mu}_{n}\right) \int\left|\psi^{(n)}\right|^{2}-\int g \overline{\psi^{(n)}} / K_{n} .
$$

Since $\int b \nabla \psi^{(n)} \overline{\psi^{(n)}}$ is purely imaginary (see (4)), we take the real part of the above relation to get

$$
\frac{1}{2} \int\left|\nabla \psi^{(n)}\right|^{2}=\tilde{\rho}_{n} \int\left|\psi^{(n)}\right|^{2}+\operatorname{Re}\left(\int g \overline{\psi^{(n)}}\right) / K_{n},
$$

taking $c \rightarrow \infty$ to get

$$
\frac{1}{2} \int\left|\nabla \psi^{*}\right|^{2} \leq \tilde{\rho} \int\left|\psi^{*}\right|^{2} .
$$

Multiplication of equations (20) with an arbitrary real $h \in H^{1}$, gives after integration and division by $c_{n}$ that

$$
\frac{1}{2 c_{n}} \int \nabla \psi^{(n)} \nabla h-\int \psi^{(n)} b \cdot \nabla h=\frac{\tilde{\rho}_{n}-i \tilde{\mu}_{n}}{c_{n}} \int \psi^{(n)} h+\frac{1}{c_{n} K_{n}} \int g h .
$$

Letting $n$ grow to infinity yields, since $\tilde{\mu}_{n} / c_{n} \rightarrow \mu$,

$$
\int \psi^{*} b \cdot \nabla h=-i \mu \int h \psi^{*} .
$$

This implies that $\psi^{*} \in H_{\mu}^{1}$. 
Now we consider an arbitrary $f \in M_{\mu}$. We multiply (20) by $\bar{f}$ to obtain after integration

$$
-\frac{1}{2} \int \nabla \psi^{(n)} \overline{\nabla f}+c_{n} \int b \nabla \psi^{(n)} \bar{f}=\left(-\tilde{\rho}_{n}+i \tilde{\mu}_{n}\right) \int \psi^{(n)} \bar{f}-\int g \bar{f} / K_{n} .
$$

Since $f \in M_{\mu}$, we have $b \cdot \nabla f=i \mu f$. Then

$$
\int b \nabla \psi^{(n)} \bar{f}=-\int \psi^{(n)} b \nabla \bar{f}=i \mu \int \psi^{(n)} \bar{f} .
$$

We obtain

$$
-\frac{1}{2} \int \nabla \psi^{(n)} \overline{\nabla f}=\left(-\tilde{\rho}_{n}+i \delta_{n}\right) \int \psi^{(n)} \bar{f}-\int g \bar{f} / K_{n} .
$$

Taking $n \rightarrow \infty$, we have

$$
-\frac{1}{2} \int \nabla \psi^{*} \overline{\nabla f}=(-\tilde{\rho}+i \delta) \int \psi^{*} \bar{f} .
$$

Since $f \in M_{\mu}, \psi^{*} \in H_{\mu}^{1}$, by Lemma 2 .

$$
\frac{1}{2} \int \nabla \psi^{*} \overline{\nabla f}=\rho_{\mu} \int \psi^{*} \bar{f}
$$

Together, we have

$$
(\epsilon-i \delta) \int \psi^{*} \bar{f}=0 .
$$

Thus, $\psi^{*} \perp M_{\mu}$. This implies $\psi^{*}=0$. Otherwise, we will get a contradiction because of the inequality (21) and Lemma 4 if $\alpha>0$ is small enough. This is however not possible, since

$$
\int\left|\psi^{*}\right|^{2}=\lim \int\left|\psi^{\left(c_{n}\right)}\right|^{2}=1 .
$$

Proof of Theorem 2. Since we ruled out the spectrum of $L_{c}$ in $B_{\alpha_{o}}\left(-\rho_{\mu}+i c \mu\right)$, we have

$$
0=\int_{\Gamma_{c}}\left(L_{c}-(-\rho+i c \mu+z)\right)^{-1} g d z \quad \text { a.s. }
$$

for $\Gamma_{c}=\left\{z=-\epsilon+i \delta ; \epsilon^{2}+\delta^{2}=\alpha_{0}^{2}\right\}$. It then follows that

$$
\begin{aligned}
\int|2 \pi i g|^{2} d \mathrm{vol} & =\int\left|\int_{\Gamma_{c}}\left(L_{c}-\left(-\rho_{\mu}+i c \mu+z\right)\right)^{-1} g d z+\int_{\Gamma_{c}} \frac{1}{z} d z g\right|^{2} d \mathrm{vol} \\
& \leq \int_{\Gamma_{c}} \int\left|\left(L_{c}-\left(-\rho_{\mu}+i c \mu+z\right)\right)^{-1} g(x)+\frac{1}{z} g(x)\right|^{2} d \operatorname{vol}(x) d z \\
& =\int_{\Gamma_{c}} \int\left|-\varphi_{-\rho_{\mu}+i c \mu+z}^{(c)}-\frac{\epsilon+i \delta}{\epsilon^{2}+\delta^{2}} g(x)\right|^{2} d \operatorname{vol}(x) d z
\end{aligned}
$$

Here we recall that $\varphi_{z}^{(c)}$ is $-\left(L_{c}-z\right)^{-1} g$ and is a function depending on $z$. We know that

$$
\int\left|\varphi_{z}^{(c)}\right|^{2} d \mathrm{vol}, z=-\epsilon+i \delta+\left(-\rho_{\mu}+i c \mu\right),
$$


is uniformly bounded for $\epsilon^{2}+\delta^{2}=\alpha_{0}^{2}$ and $c>0$ (see Lemma 11). Since $\varphi_{z}^{(c)}$ converges toward

$$
\varphi_{z}^{*}=-\frac{\epsilon+i \delta}{\epsilon^{2}+\delta^{2}} g
$$

the last term converges toward zero. This is a contradiction and the statement of the theorem follows.

\section{Some CONCLUding EXAMPLES}

3.1. Geodesic flows. The geodesic flow on a compact Riemannian manifold $(M, g)$ is defined on the tangent bundle $T M$. If we denote the local coordinates of $T M$ by $(u, \dot{u}), u \in M, \dot{u} \in T_{u} M$, then the function $E(u, \dot{u}):=\frac{1}{2} g(\dot{u}, \dot{u})$ is a first integral for the geodesic flow. The flow thus can be regarded as a flow on the sphere bundle $S M$ over $M$, which itself is a compact manifold with a canonical Riemannian structure. Since the geodesic flow conserves the natural Riemannian volume on $S M$, there exists a divergence-free vector field $b$ on $S M$, which generates the geodesic flow. We denote by $\Delta_{S M}$ the Laplace-Beltrami operator on the sphere bundle $S M$. Now we can apply the theory of the previous section to the following family of operators:

$$
L_{c} f:=\Delta_{S M} f+c b \cdot \nabla f .
$$

In the following we want to use our main result to understand the behavior of the spectral gaps of these operators for some specific examples.

Example 1. We first analyze the rather trivial example of a sphere $S^{2}$. For every point $x \in S^{2}$ the tangent space $T_{x} S^{2}$ is naturally embedded into $\mathbb{R}^{3}$. We thus can identify each element from $S M$ with a pair $(x, \dot{x})$ of orthogonal unit vectors in $\mathbb{R}^{3}$. There then exists a unique unit vector $y(x, \dot{x})$ which completes $(x, \dot{x})$ to a positively oriented orthogonal base. The resulting matrix $(x, \dot{x}, y)$ is an element from $S O(3)$, and we see that the sphere bundle $S M$ is diffeomorphic to the Lie-group $S O(3)$. Furthermore it is now easy to see that the geodesic flow acts on $S O(3)$ through left multiplication with the matrices fixing the element $y$, i.e.:

$$
\Phi_{t}=\left(\begin{array}{ccc}
\sin t & \cos t & 0 \\
-\cos t & \sin t & 0 \\
0 & 0 & 1
\end{array}\right) .
$$

This means that a geodesic corresponds to an orbit of the subgroup $S O(2)$ in $S O(3)$. If we identify the points on the geodesics, we obtain $S O(3) / S O(2)=S^{2}$ (see Gallot et al. 13]). This means that every element $y$ from $S^{2}$ determines a unique directed geodesic, which itself of course is isomorphic to an $S^{1}$. Thus all functions $f: S M \rightarrow \mathbb{R}$ can be represented as $f(x, y)$, where $x \in S^{1}$ and $y \in S^{2}$. Now, let $f$ be an $H^{1}$-eigenfunction of the operator $b \cdot \nabla$ corresponding to the eigenvalue $i \mu$. Since every geodesic has period $2 \pi$ the eigenvalue must be of the form $i k$ with $k$ integer. If we define the mean

$$
\bar{f}(y):=\frac{1}{2 \pi} \int_{S^{1}} f(x, y) \sigma(d x),
$$


it follows from the Poincaré inequality and Jensen's inequality that

$$
\begin{aligned}
\int_{S M}|\nabla f|^{2}= & \int_{S^{2}} \int_{S^{1}}\left(\left|\nabla_{x} f(x, y)\right|^{2}+\left|\nabla_{y} f(x, y)\right|^{2}\right) \sigma(d x) \sigma(d y) \\
\geq & \int_{S^{2}} \int_{S^{1}}(f(x, y)-\bar{f}(y))^{2} \sigma(d x) \sigma(d y)+2 \pi \int_{S^{2}}\left|\nabla_{y} \bar{f}(y)\right|^{2} \sigma(d y) \\
\geq & \int_{S^{2}} \int_{S^{1}}(f(x, y))^{2} \sigma(d x) \sigma(d y)-2 \pi \int_{S^{2}}(\bar{f}(y))^{2} \sigma(d y) \\
& +4 \pi \int_{S^{2}}(\bar{f}(y))^{2} \sigma(d y) \\
= & \int_{S^{2}} \int_{S^{1}}(f(x, y))^{2} \sigma(d x) \sigma(d y)+2 \pi \int_{S^{2}}(\bar{f}(y))^{2} \sigma(d y) .
\end{aligned}
$$

This becomes minimal if $\bar{f}(y)=0$ for all $y \in S^{2}$. We define the function $f(x, y):=$ $x_{1}$, where $x_{1}$ is the first coordinate of $x \in S^{1} \subset \mathbb{R}^{2}$. It is not difficult to see that

$$
\begin{aligned}
\int_{S M}|\nabla f|^{2} & =\int_{S^{2}} \int_{S^{1}}\left|\nabla_{x} f(x)\right|^{2} \sigma(d x) \\
& =\int_{0}^{2 \pi}\left|\partial_{\varphi} \cos (s)\right|^{2} d s=\int_{0}^{2 \pi}|\sin (s)|^{2} d s=1 .
\end{aligned}
$$

Thus it follows that $\lim _{c \rightarrow \infty} \rho(c)=1$. The careful reader will have noticed the fact that we did not use the property that $f$ is periodic, i.e. an eigenfunction of $b \cdot \nabla$. Thus the limit of $\rho(c)$ is equal to the first eigenvalue of the Laplacian on $S O(3)$. This is not very surprising since the geodesic flow acts as isometries on $S O(3)$. Thus the heat semigroup commutes with the flow and as a result the decay of the heat semigroup is not influenced by the drift.

Example 2. For compact surfaces with negative curvature it is well known that the geodesic flow is mixing and therefore weakly mixing (see Anosov [1]). It then follows that there exists no nonconstant eigenfunction (see Cornfeld et al. [10, p. 29). This implies that for all $\mu \in \mathbb{R}$ the spaces $H_{\mu}^{1}$ contain only the zero function. Theorem 1 then implies that $\rho(c)$ diverges to infinity as $c \rightarrow \infty$. This result also follows from the considerations in Constantin et al. [9].

Example 3. We now come to the geodesic flow on the ellipsoid. The material used in this example can be found in Klingenberg [20, p. $303 \mathrm{ff}$. $\mathbb{R}^{3}$ :

We fix $0<a_{0}<a_{1}<a_{2}$ and define the ellipsoid $\mathcal{E}$ to be the following subset of

$$
\mathcal{E}:=\left\{\frac{x_{0}^{2}}{a_{0}}+\frac{x_{1}^{2}}{a_{1}}+\frac{x_{2}^{2}}{a_{2}}=1\right\}
$$

Outside of the four umbilic points

$$
\left(x_{0}, x_{1}, x_{2}\right)=\left( \pm \frac{\sqrt{a_{0}} \sqrt{a_{1}-a_{0}}}{\sqrt{a_{2}-a_{0}}}, 0, \pm \frac{\sqrt{a_{2}} \sqrt{a_{2}-a_{1}}}{\sqrt{a_{2}-a_{0}}}\right)
$$


we can use the elliptic coordinates $u_{1}, u_{2} \in\left(a_{0}, a_{1}\right) \times\left(a_{1}, a_{2}\right)$ to parametrize the intersection of the ellipsoid $\mathcal{E}$ with the first quadrant as follows:

$$
\begin{aligned}
& x_{0}\left(u_{1}, u_{2}\right):=\sqrt{\frac{a_{0}\left(u_{1}-a_{0}\right)\left(u_{2}-a_{0}\right)}{\left(a_{1}-a_{0}\right)\left(a_{2}-a_{0}\right)}}, \\
& x_{1}\left(u_{1}, u_{2}\right):=\sqrt{\frac{a_{1}\left(u_{1}-a_{1}\right)\left(u_{2}-a_{1}\right)}{\left(a_{0}-a_{1}\right)\left(a_{2}-a_{1}\right)}}, \\
& x_{2}\left(u_{1}, u_{2}\right):=\sqrt{\frac{a_{2}\left(u_{1}-a_{2}\right)\left(u_{2}-a_{2}\right)}{\left(a_{0}-a_{2}\right)\left(a_{1}-a_{2}\right)}} .
\end{aligned}
$$

The seven other parts of the ellipsoid are then obtained by suitable reflections. If we define

$$
U_{i}\left(u_{i}\right):=(-1)^{i} \frac{u_{i}}{4\left(a_{0}-u_{i}\right)\left(a_{1}-u_{i}\right)\left(a_{2}-u_{i}\right)}, \quad i=0,1,2,
$$

then the functions

$$
F(u, \dot{u}):=\left(-u_{1}+u_{2}\right)\left(u_{2} U_{1} \dot{u}_{1}+u_{1} U_{2} \dot{u}_{2}\right)
$$

and

$$
E(u, \dot{u}):=\left(-u_{1}+u_{2}\right)\left(U_{1} \dot{u}_{1}^{2}+U_{2} \dot{u}_{2}^{2}\right)
$$

are first integrals for the geodesic flow on $T \mathcal{E}$ (see Klingenberg [20, p. 307). It turns out to be more convenient to describe the geodesic flow in terms of the cotangent space $T^{*} \mathcal{E}$ of $\mathcal{E}$. The bundle $T \mathcal{E}$ can be identified with the bundle $T^{*} \mathcal{E}$ with the help of the Riemannian metric on $\mathcal{E}$. The relation between cotangent coordinates $\left(u_{i}, \dot{u}_{i}\right)$ and tangent coordinates $\left(u_{i}, v_{i}\right)$ is given by

$$
\dot{u}_{i}=\frac{v_{i}}{\left(-u_{1}+u_{2}\right) U_{i}}, i=1,2 .
$$

The first integrals $E^{*}$ and $F^{*}$ corresponding to the energy $E$ and the function $F$ then become

$$
\begin{aligned}
& E^{*}(u, v)=\frac{1}{2\left(-u_{1}+u_{2}\right)}\left(\frac{1}{U_{1}} v_{1}^{2}+\frac{1}{U_{2}} v_{2}^{2}\right), \\
& F^{*}(u, v)=\frac{1}{\left(-u_{1}+u_{2}\right)}\left(\frac{u_{2}}{U_{1}} v_{1}^{2}+\frac{u_{1}}{U_{2}} v_{2}^{2}\right) .
\end{aligned}
$$

In the following we denote by $\operatorname{vol}_{F^{*}}$ the image measure of the Riemannian volume vol on $S \mathcal{E}$ with respect to the level-function $F^{*}$. For every fixed $\gamma \in\left(a_{0}, a_{1}\right) \cup\left(a_{1}, a_{2}\right)$ the level-set $F^{*}(u, \dot{u})=\gamma$ splits into two embedded 2-dimensional invariant tori $T_{\gamma}^{ \pm}$ for the flow. For $\gamma \in\left(a_{1}, a_{2}\right)$ the flow lines from $T_{\gamma}^{ \pm}$wind around the $x_{2}$-axis and oscillate between the lines defined by $u_{2}=\gamma$. The $+/-$ in the definition of $T_{\gamma}^{ \pm}$ indicates the direction of the winding. Similarly, for $\gamma \in\left(a_{0}, a_{1}\right)$ the flow lines from $T_{\gamma}^{ \pm}$wind around the $x_{1}$-axis and oscillate between the lines defined by $u_{1}=\gamma$ on $\mathcal{E}$ (see Klingenberg 20], p.308). In appropriate coordinates the dynamics of the flow on the invariant torus $T_{\gamma}^{ \pm}$is equivalent to the linear flow of slope $\omega_{\gamma}$ on the flat torus (see Klingenberg [20], p.310). The geodesics are periodic if and only if $\omega_{\gamma}$ is rational. The function $\gamma \mapsto \omega_{\gamma}$ is strictly decreasing on $\left(a_{0}, a_{1}\right) \cap\left(a_{1}, a_{2}\right)$ (see Klingenberg [20], p.313). We will need the following lemma.

Lemma 12. For an arbitrary $\nu \in \mathbb{R}$ the set of $\gamma$ such that the iteration $\phi_{\nu}$ is ergodic on the torus $T_{\gamma}^{ \pm}$is a zero set with respect to $\operatorname{vol}_{F^{*}}$. 
Proof. It is well known that the transformation

$$
T(x)=\left(x_{1}+\alpha_{1}(\bmod 1), x_{2}+\alpha_{2}(\bmod 1)\right)
$$

on the flat torus is ergodic if and only if $\left(1, \alpha_{1}, \alpha_{2}\right)$ are rationlly independent (see Cornfeld et al. [10], p.64). In our situation we have

$$
\alpha_{1}=\nu / \sqrt{\omega_{\gamma}^{2}+1} \text { and } \alpha_{2}=\nu / \sqrt{\omega_{\gamma}^{-2}+1} .
$$

We thus have to show that the set of $\gamma$ such that

$$
\left(1, \nu / \sqrt{\omega_{\gamma}^{2}+1}, \nu / \sqrt{\omega_{\gamma}^{-2}+1}\right) \text { are rationally dependent }
$$

is a zero set with respect to $\operatorname{vol}_{F^{*}}$. Because of the countability of the rational numbers we only need to show for two fixed arbitrary rationals $p$ and $q$ that the set of $\gamma$ satisfying the property

$$
\left(1, p \nu / \sqrt{\omega_{\gamma}^{2}+1}+q \nu / \sqrt{\omega_{\gamma}^{-2}+1}\right) \text { are rationally dependent }
$$

is a zero set with respect to $\operatorname{vol}_{F^{*}}$. We recall that the function $\gamma \mapsto \omega_{\gamma}$ is strictly monotone. It then follows that for given $p, q, r$ the cardinality of the set

$$
A_{p, q, r}:=\left\{\gamma ; p \nu / \sqrt{\omega_{\gamma}^{2}+1}+q \nu / \sqrt{\omega_{\gamma}^{-2}+1}=r\right\}
$$

is finite. This then implies that the set

$$
A:=\bigcup_{p, q, r \text { rational }} A_{p, q, r}
$$

is countable and thus a zero set, since $\operatorname{vol}_{F^{*}}$ has no atoms.

Proposition 3. For all $\mu \in \mathbb{R}$ we have that $\psi \in H_{\mu}^{1}$ implies that $\psi$ is constant on

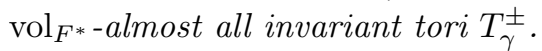

Proof. This follows from the fact that the geodesic flow on $T^{*} \mathcal{E}$ factorizes into a family of embedded invariant tori $T_{\gamma}^{ \pm}$. After a choice of suitable coordinates the restriction of the flow to those tori is equivalent to a linear flow with slope $\omega_{\gamma}$ on the flat torus. Let $m_{\gamma}^{ \pm}$denote the measure, which is induced on $T_{\gamma}^{ \pm}$through the embedding. Further, we let $\tilde{m}_{\gamma}^{ \pm}$denote the measure induced on $T_{\gamma}^{ \pm}$by its identification with the flat torus. Since the flat torus is identified with $T_{\gamma}^{ \pm}$through a coordinate-change the two measures $m_{\gamma}^{ \pm}$and $\tilde{m}_{\gamma}^{ \pm}$are equivalent. An eigenvalue $i \mu$ of $b \cdot \nabla$ corresponds to eigenfunctions $f$ which are periodic under the flow in the following sense:

$$
f \circ \phi_{2 \pi / \mu}(x)=f(x) \text { for vol-almost all } x \in S \mathcal{E} .
$$

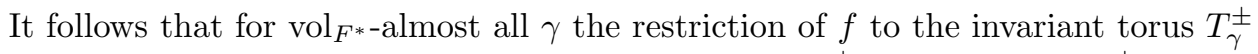
must be periodic; i.e., $f \circ \phi_{2 \pi / \mu}(x)=f(x)$ holds for $m_{\gamma}^{ \pm}$-almost all $x \in T_{\gamma}^{ \pm}$.

Moreover if $f$ is in $H^{1}$, then it is also in $L^{2}$ and its restriction to $T_{\gamma}^{ \pm}$must be in

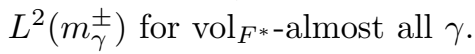

If $f$ is periodic with period $2 \pi / \mu$ with respect to the flow $\phi$, then $f$ is invariant with respect to the transformation $\phi_{2 \pi / \mu}$. In the previous lemma we saw that for $\operatorname{vol}_{F^{*}-\text { almost all } \gamma}$ the transformation $\phi_{2 \pi / \mu}$ is ergodic. This implies that for $\operatorname{vol}_{F^{*-}}$ almost all $\gamma$ the restriction of $f$ to $T_{\gamma}^{ \pm}$is $\tilde{m}_{\gamma}^{ \pm}$-almost surely constant. This finishes the proof of the proposition. 
Corollary 1. For $\mu \neq 0$ we have $H_{\mu}^{1}=\{0\}$.

The conclusion from this corollary is that in the computation of the infimum for the limiting spectral gap we can restrict our attention to the space $H_{0}$. If we take care of the fact that every pre-image of $F^{*}$ is the union of two invariant tori, we see that:

Proposition 4. $\lim _{c \rightarrow \infty} \rho(c)$ is given by

$$
\inf \left\{\frac{1}{4} \int\left(\left|\nabla\left(\varphi_{+} \circ F\right)\right|^{2}+\left|\nabla\left(\varphi_{-} \circ F\right)\right|^{2}\right) ; \int \varphi_{+}^{2} d \operatorname{vol}_{F^{*}}=\int \varphi_{-}^{2} d \operatorname{vol}_{F^{*}}=1\right\} .
$$

Proof. This follows from the previous considerations.

3.2. Three-dimensional torus. For diffusions on the $d$-dimensional torus, explicit computations can be done. These examples are very useful, since every periodic diffusion with periodic initial conditions can be related to a diffusion on a suitable torus.

Example 4. In this subsection we analyse the following three-dimensional periodic flow:

$$
\begin{aligned}
& x_{1}(t)=x_{1}(0)+t, \\
& x_{2}(t)=x_{2}(0)+\int_{0}^{t} \phi_{2}\left(x_{1}(0)+s\right) d s, \\
& x_{3}(t)=x_{3}(0)+\int_{0}^{t} \phi_{3}\left(x_{1}(0)+s\right) d s,
\end{aligned}
$$

where $\phi_{2}, \phi_{3}$ are periodic functions with period one. The corresponding vector field is given by

$$
b_{1}(x)=1, b_{2}(x)=\phi_{2}\left(x_{1}\right) \text { and } b_{3}(x)=\phi_{3}\left(x_{1}\right) .
$$

Further we assume that there exist two integers $m_{2}^{(0)}$ and $m_{3}^{(0)}$ such that

$$
\int_{0}^{1} \phi_{2}(s) d s=m_{2}^{(0)} \quad \text { and } \quad \int_{0}^{1} \phi_{3}(s) d s=m_{3}^{(0)}
$$

and that

$$
\int_{0}^{1} \phi_{2}(s) \phi_{3}(s) d s=0 .
$$

For given $0 \leq x_{1}, x_{2}, x_{3} \leq 1$ we use the fact that every flow line intersects the hyperplane $\left\{x \in \mathbb{R}^{3} \mid x_{1}=0\right\}$ in exactly one point to define the following coordinate change:

$$
\begin{aligned}
& y_{1}\left(x_{1}, x_{2}\right):=x_{2}-\int_{0}^{x_{1}} \phi_{2}(s) d s(\bmod \mathbb{Z}), \\
& y_{2}\left(x_{1}, x_{3}\right):=x_{3}-\int_{0}^{x_{1}} \phi_{3}(s) d s(\bmod \mathbb{Z}) .
\end{aligned}
$$

This new coordinate system follows the characteristics of the flow. Let $f$ be a periodic function satisfying $b \cdot \nabla f=0$. We use the invariance $f(x(t))=f(x(0))$ to 
find a function $\bar{f}:[0,1)^{2} \rightarrow \mathbb{R}$ such that

$$
f\left(x_{1}, x_{2}, x_{3}\right)=f\left(0, y_{1}, y_{2}\right)=\bar{f}\left(y_{1}, y_{2}\right) .
$$

We then have the following relations for the partial derivatives of $f$ and $\bar{f}$ :

$$
\begin{aligned}
\partial_{x_{1}} f(x) & =-\phi_{2}\left(x_{1}\right) \partial_{y_{1}} \bar{f}(y(x))-\phi_{3}\left(x_{1}\right) \partial_{y_{2}} \bar{f}(y(x)), \\
\partial_{x_{2}} f(x) & =\partial_{y_{1}} \bar{f}(y(x)) \\
\partial_{x_{3}} f(x) & =\partial_{y_{2}} \bar{f}(y(x)) .
\end{aligned}
$$

It then follows from our assumptions on $\phi_{2}$ and $\phi_{3}$ that $y_{1}\left(0, x_{2}\right)=y_{1}\left(1, x_{2}\right)$ and $y_{2}\left(0, x_{3}\right)=y_{2}\left(1, x_{3}\right)$ hold for all $x_{2}$ resp. $x_{3}$ in $[0,1]$. From this follows

$$
\begin{aligned}
\int\left(\partial_{x_{2}} f\right)^{2} & =\int_{0}^{1} \int_{0}^{1} \int_{0}^{1}\left(\frac{\partial \bar{f}}{\partial y_{1}}\right)^{2}\left(y_{2}\left(x_{1}, x_{2}\right), y_{3}\left(x_{1}, x_{3}\right)\right) d x_{2} d x_{3} d x_{1} \\
& =\int_{0}^{1} \int_{0}^{1}\left(\frac{\partial \bar{f}}{\partial y_{1}}\right)^{2}\left(y_{1}, y_{2}\right) d y_{1} d y_{2} .
\end{aligned}
$$

In the same way one has

$$
\int\left(\partial_{x_{3}} f\right)^{2}=\int_{0}^{1} \int_{0}^{1}\left(\frac{\partial \bar{f}}{\partial y_{2}}\right)^{2}\left(y_{1}, y_{2}\right) d y_{1} d y_{2} .
$$

Moreover,

$$
\begin{aligned}
\int\left(\partial_{x_{1}} f\right)^{2}= & \int\left(\phi_{2} \frac{\partial \bar{f}}{\partial y_{1}}+\phi_{3} \frac{\partial \bar{f}}{\partial y_{2}}\right)^{2} \\
= & \int_{0}^{1} \int_{0}^{1} \int_{0}^{1}\left(\phi_{2}\left(x_{1}\right) \frac{\partial \bar{f}}{\partial y_{1}}\left(y_{1}, y_{2}\right)+\phi_{3}\left(x_{1}\right) \frac{\partial \bar{f}}{\partial y_{2}}\left(y_{1}, y_{2}\right)\right)^{2} d x_{1} d y_{1} d y_{2} \\
= & \int_{0}^{1} \int_{0}^{1} \int_{0}^{1}\left(\phi_{2}\left(x_{1}\right) \frac{\partial \bar{f}}{\partial y_{1}}\right)^{2}\left(y_{1}, y_{2}\right) d x_{1} d y_{1} d y_{2} \\
& +\int_{0}^{1} \int_{0}^{1} \int_{0}^{1}\left(\phi_{3}\left(x_{1}\right) \frac{\partial \bar{f}}{\partial y_{2}}\right)^{2}\left(y_{1}, y_{2}\right) d x_{1} d y_{1} d y_{2} \\
& +2 \int_{0}^{1} \int_{0}^{1} \int_{0}^{1} \phi_{2}\left(x_{1}\right) \phi_{3}\left(x_{1}\right) \frac{\partial \bar{f}}{\partial y_{1}} \frac{\partial \bar{f}}{\partial y_{1}}\left(y_{1}, y_{2}\right) d x_{1} d y_{1} d y_{2} \\
= & \int_{0}^{1} \phi_{2}^{2}\left(x_{1}\right) d x_{1} \int_{0}^{1} \int_{0}^{1}\left(\frac{\partial \bar{f}}{\partial y_{1}}\right)^{2}\left(y_{1}, y_{2}\right) d y_{1} d y_{2} \\
& +\int_{0}^{1} \phi_{3}^{2}\left(x_{1}\right) d x_{1} \int_{0}^{1} \int_{0}^{1}\left(\frac{\partial \bar{f}}{\partial y_{2}}\right)^{2}\left(y_{1}, y_{2}\right) d y_{1} d y_{2} .
\end{aligned}
$$

Together this implies

$$
\begin{aligned}
\int|\nabla f|^{2}= & \int\left(\left(\partial_{x_{1}} f\right)^{2}+\left(\partial_{x_{2}} f\right)^{2}+\left(\partial_{x_{3}} f\right)^{2}\right) \\
= & \left(1+\int_{0}^{1} \phi_{2}^{2}\left(x_{1}\right) d x_{1}\right) \int_{0}^{1} \int_{0}^{1}\left(\frac{\partial \bar{f}}{\partial y_{1}}\right)^{2} d y_{1} d y_{2} \\
& +\left(1+\int_{0}^{1} \phi_{3}^{2}\left(x_{1}\right) d x_{1}\right) \int_{0}^{1} \int_{0}^{1}\left(\frac{\partial \bar{f}}{\partial y_{2}}\right)^{2} d y_{1} d y_{2}
\end{aligned}
$$


Example 5. We now specify a periodic vector field in the previous example. We define

$$
\phi_{2}(x):=2 \pi M \sin (2 \pi x) \text { and } \phi_{3}(x):=2 \pi M \cos (2 \pi x),
$$

where $M$ is a positive integer. The computation in the previous example shows that

$$
\begin{gathered}
\int_{0}^{1} \int_{0}^{1} \int_{0}^{1}\left(\left(\frac{\partial f}{\partial x_{1}}\right)^{2}+\left(\frac{\partial f}{\partial x_{1}}\right)^{2}+\left(\frac{\partial f}{\partial x_{1}}\right)^{2}\right) d x_{1} d x_{2} d x_{3} \\
=\left(1+2 \pi^{2} M^{2}\right) \int_{0}^{1} \int_{0}^{1}\left(\left(\frac{\partial \bar{f}}{\partial y_{1}}\right)^{2}+\left(\frac{\partial \bar{f}}{\partial y_{2}}\right)^{2}\right) d y_{1} d y_{2} .
\end{gathered}
$$

We conclude that the value of the following is $2 \pi^{2}\left(1+2 \pi^{2} M^{2}\right)$ :

$$
\inf \left\{\frac{1}{2} \int_{0}^{1} \int_{0}^{1} \int_{0}^{1}\left(\left(\frac{\partial f}{\partial x_{1}}\right)^{2}+\left(\frac{\partial f}{\partial x_{1}}\right)^{2}+\left(\frac{\partial f}{\partial x_{1}}\right)^{2}\right) d x_{1} d x_{2} d x_{3}\right\}
$$

over $f \in H_{0}^{1}$ with

$$
\begin{gathered}
\int_{0}^{1} \int_{0}^{1} \int_{0}^{1}\left|f\left(x_{1}, x_{2}, x_{3}\right)\right|^{2} d x_{1} d x_{2} d x_{3}=1 \\
\int_{0}^{1} \int_{0}^{1} \int_{0}^{1} f\left(x_{1}, x_{2}, x_{3}\right) d x_{1} d x_{2} d x_{3}=0 .
\end{gathered}
$$

Similarly, we can show that $H_{\mu}^{1} \neq\{0\}$ only when $\mu=2 k \pi$ for some $k \in Z$, and for $\mu=2 k \pi, \phi \in H_{\mu}^{1}$ if and only if

$$
\phi\left(x_{1}, x_{2}, x_{3}\right)=\exp \left(i 2 k \pi x_{1}\right) \bar{\phi}\left(y_{1}, y_{2}\right)
$$

if $y_{1}, y_{2}$ are defined by (22). The value of the following is $2 \pi^{2}\left(1+2 \pi^{2} M^{2}\right)+2 \pi^{2} k^{2}$ :

$$
\inf \left\{\frac{1}{2} \int_{0}^{1} \int_{0}^{1} \int_{0}^{1}\left(\left(\frac{\partial f}{\partial x_{1}}\right)^{2}+\left(\frac{\partial f}{\partial x_{1}}\right)^{2}+\left(\frac{\partial f}{\partial x_{1}}\right)^{2}\right) d x_{1} d x_{2} d x_{3}\right\}
$$

over $f \in H_{\mu}^{1}$ with

$$
\begin{gathered}
\int_{0}^{1} \int_{0}^{1} \int_{0}^{1}\left|f\left(x_{1}, x_{2}, x_{3}\right)\right|^{2} d x_{1} d x_{2} d x_{3}=1 \\
\int_{0}^{1} \int_{0}^{1} \int_{0}^{1} f\left(x_{1}, x_{2}, x_{3}\right) d x_{1} d x_{2} d x_{3}=0
\end{gathered}
$$

That is,

$$
\rho_{\mu}=2 \pi^{2}\left(1+2 \pi^{2} M^{2}\right)+2 \pi^{2} k^{2}, \mu=2 k \pi, k \in Z .
$$

We conclude that the limit of $\rho(c)(c \rightarrow \infty)$ is $2 \pi^{2}\left(1+2 \pi^{2} M^{2}\right)$.

\section{ACKNOWLEDGEMENTS}

The first author wants to thank the staff and the probability group from Academia Sinica for the constant support during his visit to Taiwan. We would 
like to thank the referee for the useful suggestions to improve the presentation of the paper.

\section{REFERENCES}

1. D. Anosov, Geodesic flows on closed Riemannian manifolds of negative curvature, Proceedings of the Steklov Institute of Mathematics, 90, 1967. MR.0224110 (36:7157)

2. H. Aref, The development of chaotic advection, Phys. Fluids, 14 (2002), 1315-1325. MR:1900232 (2003d:76085)

3. H. Berestycki, F. Hamel and N. Nadirashvili, Elliptic eigenvalue problems with large drift and applications to nonlinear propagation phenomena, Commun. Math. Phys., 253 (2005), 451-480. MR2140256 (2006b:35057)

4. R.N. Bhattacharya,V.K. Gupta and H.F. Walker, Asymptotics of solute dispersion in periodic porous media, SIAM J. Appl. Math., 49 (1989), 86-98. MR978827 (90f:35113)

5. S. Brooks, Markov chain Monte Carlo method and applications, The Statistician, 47 (1998) 69-100.

6. M.F. Chen, Eigenvalues, Inequalities, and Ergodic Theory, Springer-Verlag, London, 2005. MR 2105651 (2005m:60001)

7. M.F. Chen and F.Y. Wang, Estimation of the first eigenvalue of second-order elliptic operators, J. Funct. Anal., 131 (1995), 345-363. MR1345035 (96g:35142)

8. M.F. Chen and F.Y. Wang, Estimation of spectral gap for elliptic operators, Trans. Amer. Math. Soc., 349 (1997), 1239-1267. MR1401516 (97h:35175)

9. P. Constantin, A. Kiselev, L. Ryzhik and A. Zlatos, Diffusion and mixing in fluid flow, Ann. Math. (2), 168 (2008), 643-674. MR.2434887

10. I. P. Cornfeld, S. V. Fomin and Ya. Sinai, Ergodic Theory, Grundlehren der mathematischen Wissenschaften, 245, Springer-Verlag Berlin, 1982. MR832433 (87f:28019)

11. A. Fannjiang and G. Papanicolaou, Convection enhanced diffusions for periodic flows, SIAM J. Appl. Math., 54(1994), 333-408. MR1265233 (95d:76109)

12. B. Franke, Integral inequalities for the fundamental solutions of diffusions on manifolds with divergence-free drift, Math. Z., 246(2004), 373-403. MR2031461 (2005e:58060)

13. S. Gallot, D. Hulin and J. Lafontaine, Riemannian geometry, Universitext, Springer-Verlag, Berlin, 1987. MR909697 (88k:53001)

14. W.K. Hastings, Monte Carlo sampling methods using Markov chains and their applications, Biometrica, 57 (1970), 97-109.

15. S. Heinze, Diffusion-advection in cellular flows with large Peclet numbers, Arch. Rat. Mech. Anal., 168 (2003), 329-342. MR1994746 (2004e:76037)

16. C.R. Hwang, S.Y. Hwang-Ma and S.J. Sheu, Accelerating Gaussian diffusions, Ann. Appl. Prob., 3 (1993), 897-913. MR1233633 (95c:60071)

17. C.R. Hwang, S.Y. Hwang-Ma and S.J. Sheu, Accelerating diffusions, Ann. Appl. Prob., 15(2005), 1433-1444. MR2134109 (2006e:60113)

18. C.R. Hwang and H. M. Pai, Blowing up spectral gap of Laplacian on $N$-torus by antisymmetric perturbations, preprint, (2006).

19. S. Kirkpatrick, C.D. Gelatt and M.P. Vecchi, Optimization by simulated annealing, Science, 220 (1983), 671-680. MR.702485 (85f:90091)

20. W. Klingenberg, Riemannian geometry, de Gruyter Studies in Mathematics, Walter de Gruyter and Co., Berlin-New York, (1982). MR666697 (84j:53001)

21. H. J. Kushner, Asymptotic global behavior for stochastic approximation and diffusions with slowly decreasing noise effects: Global minimization via Monte Carlo, SIAM J. Appl. Math., 47 (1987), 169-185. MR.873242 (88k:60049)

22. P. Li and S.T. Yau, Estimates of eigenvalues of a compact Riemannian manifold, Proc. Sympos. Pure Math., 36, Amer. Math. Soc., 1980, 205-240. MR.573435 (81i:58050)

23. D. Marquez, Convergence rates for annealing diffusion processes, Ann. Appl. Prob., 7 (1997) 1118-1130. MR $1484800(99 \mathrm{~b}: 65006)$

24. N. Metropolis and S. Ulam, A property of randomness of arithmetical functions, Amer. Math. Monthly, 60(1953), 252-253. MR0053416 (14:770g)

25. J.M. Ottino, Mixing, chaotic advection, and turbulence, Annual Rev. Fluid Mech., 22 (1990), 207-253. MR.1043921 (91e:76067) 
26. R. D. Richtmyer, Principles of Advanced Mathematical Physics, Vol. 1, Texts and Monographs in Physics, Springer-Verlag, New York, 1978. MR.517399 (84h:00024a)

27. B. Shraiman and E. Siggia, Scalar turbulence, Nature, 405(2000), 639-646.

28. S. Wiggins and J.M. Ottino, Foundations of chaotic mixing, Phil. Trans. R. Soc. Lond. A, 362(2004), 937-970. MR2107642(2006b:37012)

Fakultät für Mathematik, Ruhr-Universität Bochum, Universitätsstrasse 150, 44780 Bochum, Germany

E-mail address: Brice.Franke@ruhr-uni-bochum.de

Institute of Mathematics, Academia Sinica, Nankang, Taipei 11529, Taiwan

E-mail address: crhwang@math.sinica.edu.tw

Department of Statistics, National Taipei University, No. 151, University Rd., San Shia, TAIPEI 237, TAIWAN

E-mail address: hpai@mail.ntpu.edu.tw

Institute of Mathematics, Academia Sinica, Nankang, Taipei 11529, Taiwan

E-mail address: sheusj@math.sinica.edu.tw 